9. Shah AH, Osten M, Leventhal A, et al. Percutaneous intervention to treat platypnea-orthodeoxia syndrome: The Toronto experience. JACC Cardiovasc Interv. 2016;9:1928-38.

Esther González-Bartol ${ }^{a, *}$, Antonio Rojas-González ${ }^{a}$, Pablo Díez-Villanueva ${ }^{\mathrm{a}}$, Carmen Acosta-Gutiérrez ${ }^{\mathrm{b}}$, Eduardo Pozo-Osinalde ${ }^{a}$ y Fernando Alfonso ${ }^{a}$

a Servicio de Cardiología, Hospital Universitario La Princesa, Madrid, España

b Servicio de Neumología, Hospital Universitario La Princesa, Madrid, España
* Autor para correspondencia. C/ Diego de León, 62, 28006 Madrid, España; Teléfono: +34915202272;

Fax: +34 915202268 .

Correo electrónico: esthergonzba@gmail.com

(E. González-Bartol).

http://dx.doi.org/10.1016/j.acmx.2016.12.005 1405-9940/

(C) 2016 Instituto Nacional de Cardiología Ignacio Chávez. Publicado por Masson Doyma México S.A. Este es un artículo Open Access bajo la licencia CC BY-NC-ND (http:// creativecommons.org/ licenses/by-nc-nd/4.0/).

\section{Patrón de Brugada desenmascarado por propafenona en un hombre de 84 años}

\section{Brugada pattern unmasked by propafenone in an 84-years-old man}

\section{Introducción}

El síndrome de Brugada es una canalopatía hereditaria que fue descrita por primera vez en $1992^{1}$. El síndrome puede tener 3 tipos de expresiones electrocardiográficas. La del tipo ।, que es la que tiene valor diagnóstico específico, es la elevación descendente del ST mayor a $2 \mathrm{~mm}$ en precordiales derechas en un electrocardiograma de superficie de 12 derivadas (V1 a V3) con ondas $\mathrm{T}$ negativas. Existen 2 patrones electrocardiográficos más, que aunque deben hacer sospechar la canalopatía, carecen de un valor preciso en el diagnóstico. Otra característica del síndrome es la predisposición para tener arritmias ventriculares como taquicardia ventricular polimórfica o fibrilación ventricular ${ }^{2,3}$.

De acuerdo a los diversos consensos ${ }^{4}$, el síndrome de Brugada solo se puede diagnosticar en presencia de un electrocardiograma de tipo 1 , combinado con fibrilación o taquicardia ventricular documentada, inducción de arritmias en un estudio electrofisiológico, síncope o respiración agónica nocturna, historia familiar de muerte súbita antes de los 45 años o patrón electrocardiográfico de tipo । en otros miembros de la familia ${ }^{4}$.

En años recientes se ha avanzado mucho en la comprensión del síndrome. Se han identificado múltiples mutaciones ligadas a su expresión clínica que comprenden anomalías funcionales de los canales de sodio, potasio y calcio. La mayor concentración de canales $I_{\text {to }}$ en el epicardio ventricular derecho, en presencia de la canalopatía, genera gradientes eléctricos transmurales que inducen una reentrada en fase 2 y originan las taquicardias ventriculares ${ }^{3-5}$.

La mayoría de los pacientes tiene síntomas como palpitaciones o síncope, pero la forma más grave de presentación es la muerte súbita, que predomina entre los 30 y 50 años y en el género masculino, con una relación de 8-10:1. La revisión de casos rescatados de paro cardiaco o de los pacientes con el diagnóstico muestra que los enfermos refieren palpitaciones o síncope y que hay otras anomalías electrocardiográficas como un segmento PR prolongado o arritmias supraventriculares como fibrilación auricular.

\section{Presentación del caso}

Se trata de un hombre de 84 años de edad, sin antecedentes familiares relevantes y sin historia de muerte súbita en la familia. El paciente tiene hiperplasia prostática benigna que requirió de resección transuretral de próstata 6.5 años antes de la presente evaluación. Debido a recidiva de molestias obstructivas urinarias asociadas, se le realiza una valoración preoperatoria de rutina en su ciudad y se obtiene un electrocardiograma en reposo (fig. 1). Aparentemente se le encuentran extrasístoles supraventriculares (ESV) aisladas y se le realiza un Holter de $24 \mathrm{~h}$. Se reporta una frecuencia cardiaca promedio de 53 latidos por minuto (lpm) con 1,968 ESV aisladas, sin carreras de taquicardia paroxística supraventricular o arritmias complejas. Se le administra propafenona $150 \mathrm{mg} 2$ veces al día y se pospone la cirugía.

Dos meses más tarde, el paciente menciona lasitud, disnea de medianos esfuerzos, palpitaciones, mareos frecuentes y lipotimias sin llegar a tener síncope. Se realiza un nuevo Holter que muestra una frecuencia cardiaca promedio de 52 lpm, con 735 ESV, 114 extrasístoles ventriculares y periodos de bloqueo auriculoventricular completo con disociación AV. Con este resultado se le propone un implante de marcapasos, y el paciente decide pedir una segunda opinión, por lo que acude a este centro.

La exploración física es irrelevante desde el punto de vista cardiovascular, sin embargo, en el electrocardiograma se identifica un patrón de Brugada tipo I (fig. 2) con bloqueo AV de primer grado. Se decide suspender propafenona y dejarlo sin tratamiento antiarrítmico. Tres semanas después se realiza nuevo Holter, en el que se encuentra una frecuencia cardiaca promedio de 57 lpm, con 591 ESV. El estudio muestra conducción auriculoventricular normal durante todo el registro y ausencia de pausas sinusales superiores a $2 \mathrm{seg}$. En la figura 3 se muestran los electrocardiogramas seriados del paciente, específicamente las derivaciones V1 a V3. El panel 3A muestra el ECG basal antes de que el enfermo recibiese propafenona $(08 / 08 / 13)$. El panel 3B (14/11/13) muestra el ECG obtenido al revisar 


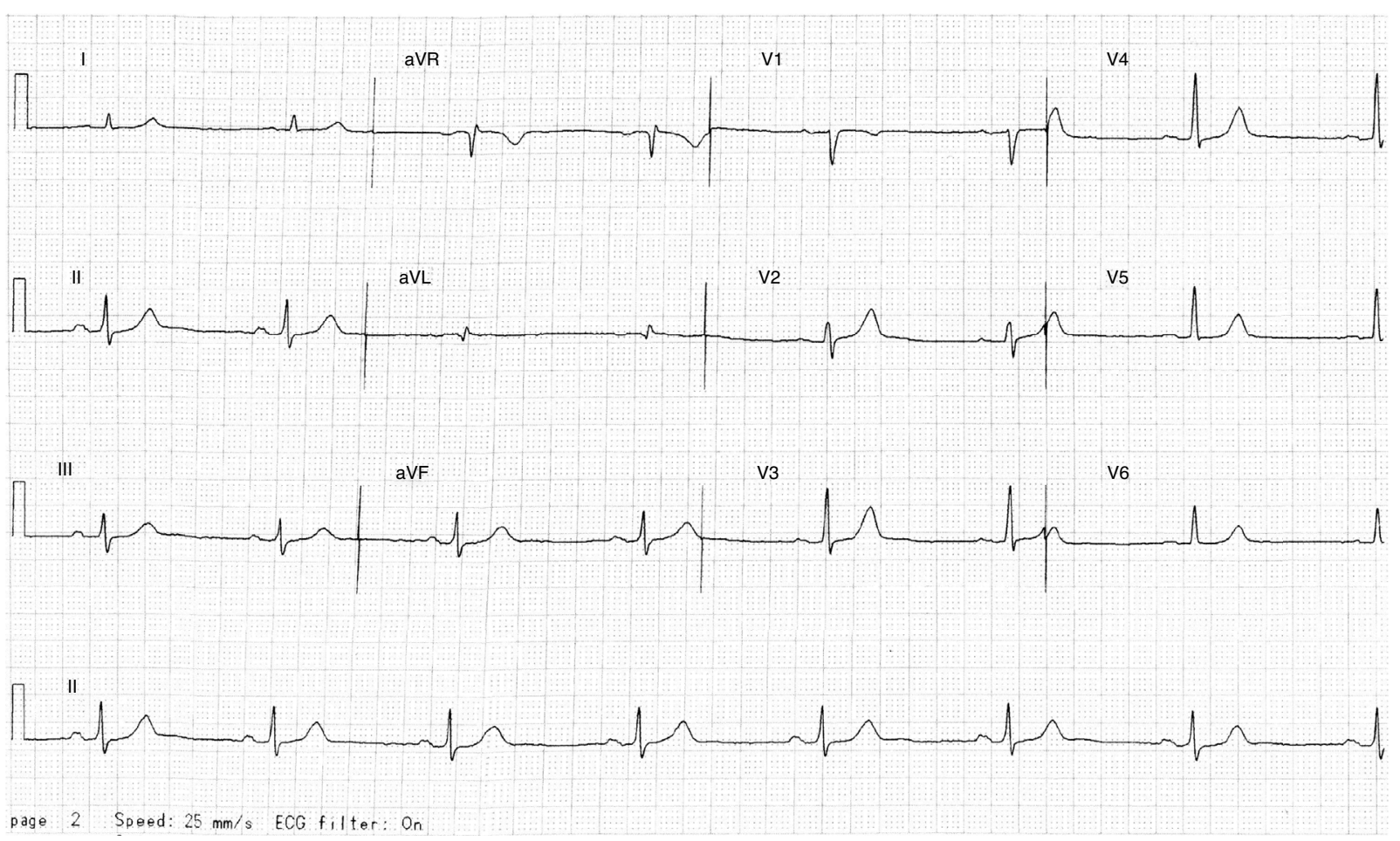

Figura 1 Electrocardiograma basal preoperatorio.

al enfermo con efecto de propafenona, en el que se aprecia un patrón de Brugada tipo I (elevación del ST de V1 a V3 con morfología de bloqueo de rama derecha). Finalmente, el panel 3C (05/12/13) muestra el ECG después de suspender propafenona. Ahí se aprecia un retorno al aspecto normal previo a la administración del fármaco. El paciente se mantuvo asintomático a lo largo de un seguimiento de 18 meses.

\section{Discusión}

La prevalencia del síndrome de Brugada en población abierta va del 0 al $0.3 \%$ en sujetos estudiados por diversas razones (adquisición de un seguro médico, pruebas de escrutinio de salud cardiovascular, revisiones de salud general) ${ }^{6}$. Por otro lado, las poblaciones de alto riesgo descritas inicialmente

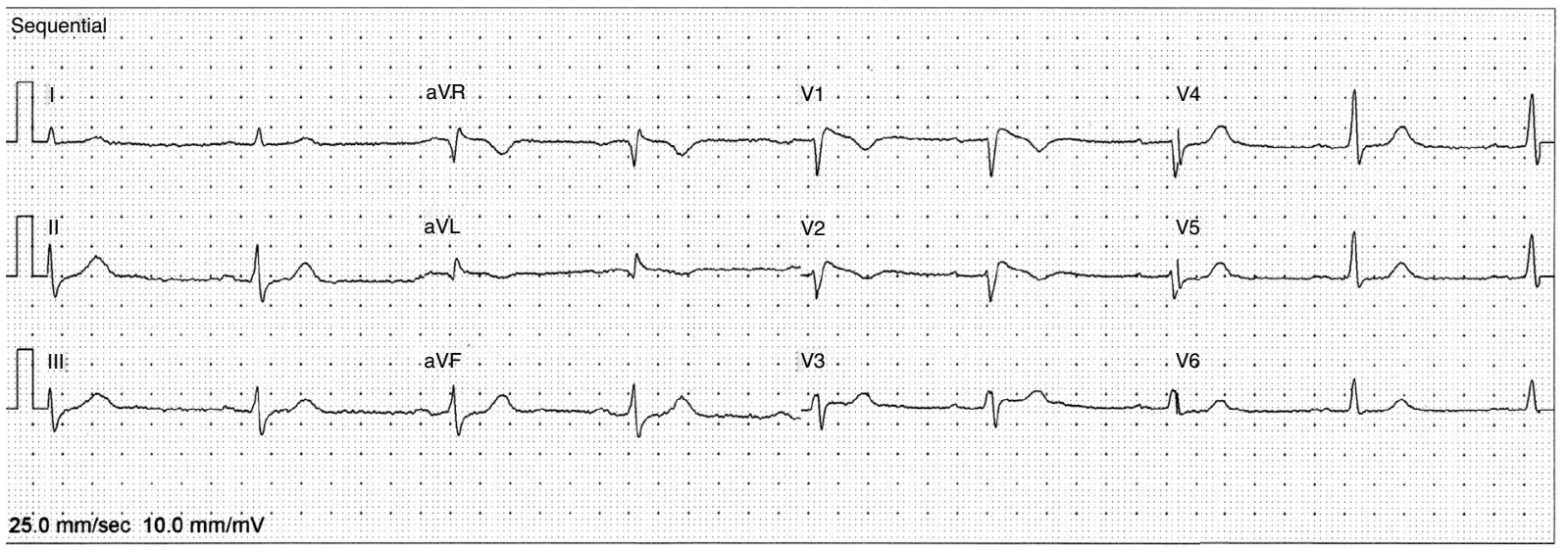

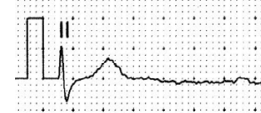

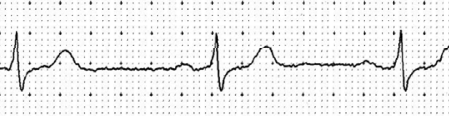

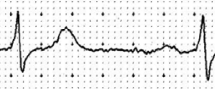

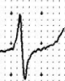<smiles>CCCCCC</smiles>

Figura 2 Electrocardiograma con patrón de Brugada. 

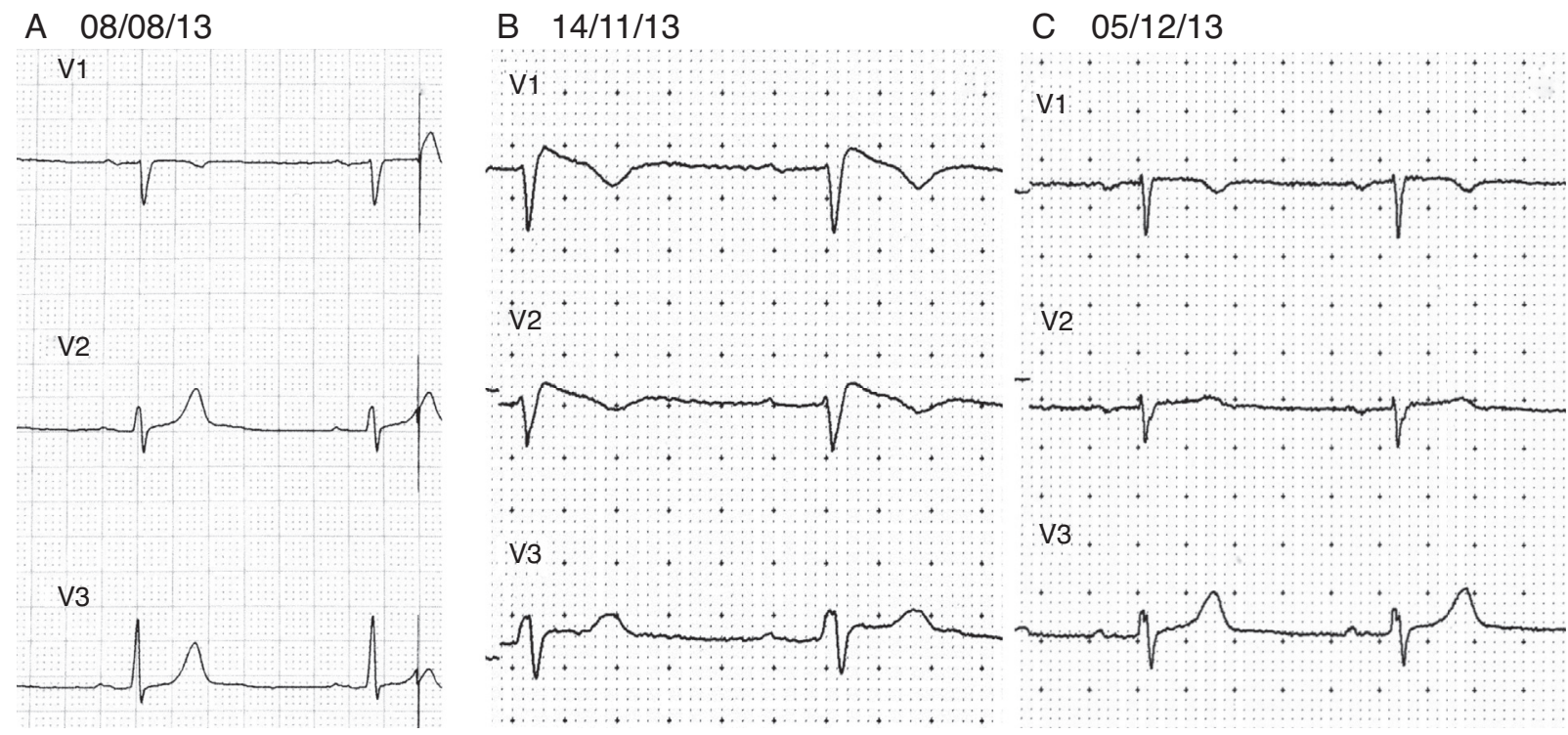

V3
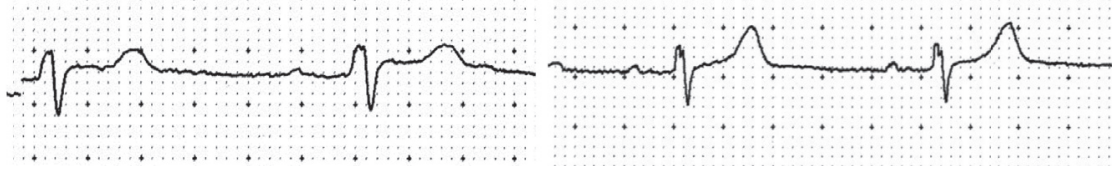

Figura 3 Electrocardiogramas (ECG) seriados del paciente. En el panel 3A se encuentra el ECG «basal» del 08/08/13. El panel 3B muestra el ECG del 14/11/13 con efecto de propafenona y patrón de Brugada tipo I. El panel 3C muestra el ECG del 05/12/13 después de suspender propafenona, con retorno al aspecto normal previo a la administración del fármaco.

corresponden a hombres jóvenes entre la tercera y quinta décadas de la vida ${ }^{7}$.

Debido al riesgo de muerte súbita que conlleva el síndrome, se ha intentado establecer un sistema que permita identificar a los sujetos de alto riesgo, sin embargo todos los esquemas tienen limitaciones, lo que obliga a individualizar los planes de evaluación y estratificación de riesgo.

La presencia espontánea de un electrocardiograma de tipo I en pacientes rescatados de muerte súbita o con síncopes supone un criterio de alto riesgo e indicación para el implante de un desfibrilador automático implantable. Sin embargo, hay un amplio espectro de pacientes que pueden tener patrones electrocardiográficos cambiantes o una historia clínica poco clara en los que es necesario recurrir a diferentes pruebas.

Dentro de estas, están las de reto farmacológico con antiarrítmicos de clase I. La propafenona es una de las opciones usadas para desenmascarar los cambios electrocardiográficos, aunque no se recomienda de forma rutinaria debido a que la vida media del fármaco es larga y ello pone al paciente en riesgo de tener arritmias ventriculares graves durante más tiempo. Sin embargo, existen reportes en donde se ha administrado propafenona por otras razones y se ha desenmascarado la canalopatía $a^{8-10}$.

La particularidad del caso que se expone es que la anomalía se presentó a una edad avanzada y como consecuencia de un tratamiento antiarrítmico que podría ser discutido. Aunque hay un caso reportado de 84 años, es poco común que la canalopatía se manifieste a esta edad. Un reciente estudio menciona que de 437 pacientes, solo 25 eran mayores de 70 años, y en ellos el pronóstico del síndrome es favorable, prácticamente sin arritmias ventriculares durante el seguimiento, sin embargo se enfatiza la importancia de revisar a la familia ${ }^{11}$. En el presente caso, 2 de sus 5 hijos accedieron a ser estudiados y mostraron electrocardiogramas normales.

En el caso que se presenta, la canalopatía solo se manifestó cuando se administró un antiarrítmico de clase IC en una persona de 84 años, por lo que el riesgo real de una arritmia ventricular grave es bajo. Los consensos sugieren que un paciente de bajo riesgo no es un candidato adecuado para recibir un desfibrilador automático implantable. En este contexto es importante diferenciar entre un «patrón electrocardiográfico de Brugada» $y$ un «síndrome de Brugada». El paciente que se presenta cumplía con los criterios ECG, sin otras manifestaciones del síndrome. Ello no quiere decir que no haya riesgo de tener arritmias mientras dure el efecto farmacológico, pero como ocurre en algunas de las fenocopias del síndrome, al eliminar la causa, se recupera el ECG normal y se reduce el riesgo de tener arritmias graves. En este caso, la utilidad de un desfibrilador implantable es limitada tanto por la edad del paciente, que alcanzó libre de síntomas, como porque los cambios ECG son inducidos por un medicamento cuyo efecto es reversible.

Actualmente se sabe que los episodios febriles y diversas condiciones pueden ser disparadores de las arritmias en el síndrome de Brugada. El mero hecho de que el paciente haya alcanzado la edad actual, lo sitúa como un sujeto de bajo riesgo.

Pese a ello, es importante mencionar que la población geriátrica debe ser estudiada y manejada con cuidado. Este paciente había recibido un tratamiento antiarrítmico para una anomalía de bajo riesgo y asintomática que a priori no justificaba esa intervención. Cuando se le evalúa posteriormente y durante el tratamiento, el paciente presenta complicaciones evitables, como el bloqueo auriculoventricular y la presencia del patrón de Brugada, que cambian su perfil de riesgo. Se puede cuestionar la presencia de una enfermedad binodal puesta de manifiesto por el medicamento y con ello la utilidad de un marcapasos. Por otro lado, si el empleo del antiarrítmico es imprescindible, entonces debería hacerse una selección muy cuidadosa del dispositivo a emplear. El marcapasos podría corregir las bradiarritmias, pero no protege al paciente en caso de una taquicardia ventricular. La utilidad del desfibrilador 
automático implantable en el contexto de este paciente debe ser evaluada con mucho cuidado, porque finalmente el elemento de riesgo lo impone una conducta iatrogénica.

Se debe enfatizar la importancia de dar un seguimiento adecuado a cualquier paciente con tratamiento antiarrítmico en vista de que, además de los efectos colaterales propios del medicamento empleado, estos pueden desenmascarar otras condiciones, y esto es independiente de la edad del sujeto. También se debe cuestionar la utilidad de los tratamientos farmacológicos en contextos específicos y, de acuerdo a ello, dar preferencia a tratamientos intervencionistas como ablación.

En el síndrome de Brugada, la ablación empieza a ser una opción terapéutica en casos seleccionados, sin embargo, este tema se sale de los alcances del presente caso.

En conclusión, las canalopatías pueden expresarse de diferentes maneras y a diferentes edades, en este caso, un patrón de Brugada manifestado por un tratamiento antiarrítmico pone a un paciente geriátrico en riesgo de desarrollar complicaciones serias. El presente caso enfatiza la necesidad de evaluar con cuidado la pertinencia de los tratamientos farmacológicos antiarrítmicos en poblaciones determinadas y la importancia de darles un seguimiento apropiado que limite los posibles riesgos asociados tanto al tratamiento en sí como a sus efectos colaterales.

\section{Bibliografía}

1. Brugada P, Brugada J. Right bundle branch block, persistent ST segment elevation and sudden cardiac death: A distinct clinical and electrocardiographic syndrome. A multicenter report. J Am Coll Cardiol. 1992;20:1391-6.

2. Asensio E, Álvarez B, Lozano E, et al. Bloqueo de rama derecha, elevación del ST y muerte súbita: Síndrome de Brugada. Arch Inst Cardiol Mex. 2000;70:301-11.

3. Benito B, Brugada J, Brugada R, et al. Síndrome de Brugada. Rev Esp Cardiol. 2009;62:1297-315.

4. Antzelevitch C, Brugada P, Borgreffe M, et al. Brugada syndrome: Report of the second consensus conference. Endorsed by the Heart Rhythm Society and the european Heart Rhythm Association. Circulation. 2005;111:659-70.

5. Martini B, Martini N, Dorantes M, et al. Pistas de una enfermedad orgánica subyacente en el síndrome de Brugada. Arch Cardiol Mex. 2017;87:49-60.

6. Pecini R, Cedergreen P, Theilade S, et al. The prevalence and relevance of the Brugada-type electrocardiogram in the Danish general population: Data from the Copenhagen City Heart Study. Europace. 2010;12:982-6.

7. Veerakul G, Nademanee K. Brugada syndrome, two decades of progress. Circ J. 2012;76:2713-22.

8. Dragios TD, Manolis AG, Kyriakides ZS. Likelihood of Brugada ECG pattern confirmed after propafenone administration for atrial fibrillation cardioversion. Acta Cardiol. 2011;66: 399-400.

9. Hasdemir C, Olukman M, Ulucan C, et al. Brugada-type ECG pattern and extreme QRS complex widening with propafenone overdose. J Cardiovasc Electrophysiol. 2006;17:565-6.

10. Guevara M, Iturralde P, de Micheli A, et al. Desenmascaramiento del síndrome de Brugada con propafenona endovenosa. Arch Cardiol Mex. 2002;72:45-8.

11. Conte G, Asmundis DE, Sieira J, et al. Clinical characteristics, management and prognosis of elderly patients with Brugada syndrome. J Cardiovasc Electrophysiol. 2014;25:514-9, http://dx.doi.org/10.1111/jce.12359.

\section{Enrique Asensio Lafuente*}

\section{División de Medicina Interna, Hospital $H+$ Querétaro, Querétaro, México}

* Hospital H + Querétaro Prol. Priv. Ignacio Zaragoza 16-H, cons 606 Querétaro, Qro. CP 76030.

Correo electrónico: easensiol@gmail.com

http://dx.doi.org/10.1016/j.acmx.2017.06.005

1405-9940/

(c) 2017 Instituto Nacional de Cardiología Ignacio Chávez. Publicado por Masson Doyma México S.A. Este es un artículo Open Access bajo la licencia CC BY-NC-ND (http: / /creativecommons.org/

licenses/by-nc-nd/4.0/). 